This is the author's final, peer-reviewed manuscript as accepted for publication. The publisher-formatted version may be available through the publisher's web site or your institution's library.

\title{
Elucidating isotopic effects in intense ultrafast laser-driven $\mathrm{D}_{2} \mathrm{H}^{+}$fragmentation
}

A. M. Sayler, J. McKenna, B. Gaire, Nora G. Kling, K. D. Carnes, B. D. Esry, I. BenItzhak

\section{How to cite this manuscript}

If you make reference to this version of the manuscript, use the following information:

Sayler, A. M., McKenna, J., Gaire, B., Kling, N. G., Carnes, K. D., Esry, B. D., \& BenItzhak, I. (2014). Elucidating isotopic effects in intense ultrafast laser-driven $\mathrm{D}_{2} \mathrm{H}^{+}$ fragmentation. Retrieved from http://krex.ksu.edu

\section{Published Version Information}

Citation: Sayler, A. M., McKenna, J., Gaire, B., Kling, N. G., Carnes, K. D., Esry, B. D., \& Ben-Itzhak, I. (2014). Elucidating isotopic effects in intense ultrafast laser-driven $\mathrm{D}_{2} \mathrm{H}^{+}$ fragmentation. Journal of Physics B: Atomic, Molecular and Optical Physics, 47(3), 031001.

Copyright: (C) 2014 IOP Publishing Ltd

Digital Object Identifier (DOI): doi:10.1088/0953-4075/47/3/031001

Publisher's Link: http://iopscience.iop.org/0953-4075/47/3/031001/

This item was retrieved from the K-State Research Exchange (K-REx), the institutional repository of Kansas State University. K-REx is available at http://krex.ksu.edu 


\title{
Elucidating isotopic effects in intense ultrafast laser-driven $\mathrm{D}_{2} \mathrm{H}^{+}$fragmentation
}

\author{
A. M. Sayler, J. McKenna, B. Gaire, Nora G. Kling, K. D. \\ Carnes, B. D. Esry, I. Ben-Itzhak \\ J.R. Macdonald Laboratory, Physics Department, Kansas State University, \\ Manhattan, Kansas 66506, USA \\ E-mail: ibi@ksu.phys.edu
}

\begin{abstract}
The triatomic hydrogen molecular ion is instrumental as a benchmark toward understanding the strong-field dynamics of polyatomic molecules. Using a crossed-beams coincidence three-dimensional momentum imaging method, we demonstrate clear isotopic effects in the fragmentation of $\mathrm{D}_{2} \mathrm{H}^{+}$induced by $7 \mathrm{fs}$ (40 fs), $790 \mathrm{~nm}$ laser pulses at an intensity of $10^{16} \mathrm{~W} / \mathrm{cm}^{2}\left(5 \times 10^{15} \mathrm{~W} / \mathrm{cm}^{2}\right)$. Our experiment uniquely separates all fragmentation channels and provides kinematically complete information for the nuclear fragments. For example, we show that for dissociative ionization of $\mathrm{D}_{2} \mathrm{H}^{+}$there is a large difference in branching ratios of the two-body channels, namely, $\mathrm{H}^{+}+\mathrm{D}_{2}^{+}$dominates $\mathrm{D}^{+}+\mathrm{HD}^{+}$, whereas there is minimal difference in branching ratios between the dissociation channels $\mathrm{H}^{+}+\mathrm{D}_{2}$ and $\mathrm{D}^{+}+\mathrm{HD}$.

PACS numbers: $33.80 . \mathrm{Wz}, 42.50 . \mathrm{Hz}$
\end{abstract}

Submitted to: J. Phys. B: At. Mol. Phys.

The triatomic hydrogen molecular ion, $\mathrm{H}_{3}^{+}$, is one of the simplest, yet most exciting molecules. It plays a major role in astrophysical chemistry $[1,2,3]$, particularly in interstellar clouds $[4,5,6,7]$, and is an important component in the chemistry of planetary atmospheres [8]. Additionally, since it is the simplest stable polyatomic, it serves as a benchmark for first principles quantum mechanical calculations [9]. These important roles have motivated many laboratory measurements of this system, in particular infrared spectroscopy $[2,10,11]$ and dissociative recombination experiments $[12,13,14,15,16,17]$. Thus, much information has already been compiled on this important system and its interactions.

Our interest in $\mathrm{H}_{3}^{+}$stems from the important role it may play in understanding the dynamics of polyatomic molecules in intense laser fields. While there have been several experimental studies of $\mathrm{H}_{3}^{+}$ejection from larger polyatomic systems arising from bond rearrangement in the intense laser field (e.g. [18, 19]), it is only recently that the first direct measurements of $\mathrm{D}_{3}^{+}$strong-field dynamics have been carried 
out, by ourselves [20, 21, 22, 23, 24] and Alexander et al. [25]. Due to its basic structure, measurements of $\mathrm{H}_{3}^{+}$can provide a benchmark for theory - and ultimately help develop the theoretical tools needed to explore larger systems, as exemplified by Lötstedt et al. [26, 27]. Further theoretical studies of the $\mathrm{H}_{3}^{+}$system can be found in Refs. [28, 29, 30, 31, 32, 33, 34, 35, 36, 37].

Interesting variants of $\mathrm{H}_{3}^{+}$and $\mathrm{D}_{3}^{+}$are their asymmetric isotopologues, $\mathrm{H}_{2} \mathrm{D}^{+}$and $\mathrm{D}_{2} \mathrm{H}^{+}$. In the Born-Oppenheimer approximation, and neglecting relativistic effects, the potential energy surfaces of $\mathrm{H}_{2} \mathrm{D}^{+}$and $\mathrm{D}_{2} \mathrm{H}^{+}$are identical to those of $\mathrm{H}_{3}^{+}$and $\mathrm{D}_{3}^{+}$. However, because of the mass difference of $\mathrm{H}$ and $\mathrm{D}$ the symmetries of the molecules are broken - thereby excluding $D_{3 h}$ symmetry and leaving only $C_{2 v}$ symmetry. Moreover, the centre of mass of $\mathrm{H}_{2} \mathrm{D}^{+}$and $\mathrm{D}_{2} \mathrm{H}^{+}$is offset from the centre of charge creating a permanent dipole moment that is absent in $\mathrm{H}_{3}^{+}$and $\mathrm{D}_{3}^{+}$. In principle this in itself may lead to differing behaviour of these systems, as an intense laser may drive additional permanent dipole transitions that are absent from homonuclear molecules, although usually they elude experimental observation (see discussion in Ref. [38]).

Arguably more important, the asymmetry of $\mathrm{H}_{2} \mathrm{D}^{+}$and $\mathrm{D}_{2} \mathrm{H}^{+}$can also lead to isotopic effects. For example, $\mathrm{D}_{2} \mathrm{H}^{+}$when singly ionized may undergo two-body fragmentation into $\mathrm{H}^{+}+\mathrm{D}_{2}^{+}$or $\mathrm{D}^{+}+\mathrm{HD}^{+}$. Should the branching ratio of these channels differ, i.e., one channel dominates over the other, we refer to this as an isotopic effect. In this communication, we explore isotopic effects in the strong-field fragmentation of $\mathrm{D}_{2} \mathrm{H}^{+}$ by addressing questions such as 'is there a preference for breakup into $\mathrm{H}^{+}+\mathrm{D}^{+}+\mathrm{D}(1 s)$ over $\mathrm{D}^{+}+\mathrm{D}^{+}+\mathrm{H}(1 s)$ ?'. To our knowledge, we observe the first clear experimental evidence of such an isotopic effect in an intense laser, where the thresholds of the two channels are almost the same (within $3.7 \mathrm{meV}$ of each other due to the small binding energy difference of $\mathrm{H}(1 s)$ and $\mathrm{D}(1 s)$ ) — see note [39]. In addition, we compare the kinetic energy release (KER) from different channels, in particular $\mathrm{H}^{+}+\mathrm{D}^{+}+\mathrm{D}$ and $\mathrm{D}^{+}+\mathrm{D}^{+}+\mathrm{H}$, and also find differences in their energetics.

Recently, interest in isotopic effects in $\mathrm{D}_{2} \mathrm{H}$ and $\mathrm{D}_{2} \mathrm{H}^{+}$have emerged in other research areas, particularly electron-impact studies. For the dissociative recombination of $\mathrm{D}_{2} \mathrm{H}^{+}$, Buhr et al. [40] have reported a preference for fragmentation to the $\mathrm{H}+\mathrm{D}_{2}$ channel over the $\mathrm{D}+\mathrm{HD}$ channel. They observed a ratio for $2\left[\mathrm{H}+\mathrm{D}_{2}\right] /[\mathrm{D}+\mathrm{HD}]$ ranging from $1.27 \pm 0.05$ to $3.7 \pm 0.5$ for relative electron-ion energies in the range 0 to $5 \mathrm{eV}$. Note that a ratio of 1 corresponds to no isotopic effect (see note [41]). Defrance et al. explored electron-impact dissociation and single ionization of $\mathrm{D}_{2} \mathrm{H}^{+}$[42], reporting almost no difference in branching ratio between the $\mathrm{H}^{+}+\mathrm{D}_{2}$ and $\mathrm{D}^{+}+\mathrm{HD}$ channels, but significant isotopic effects for $2\left[\mathrm{D}_{2}^{+}+\mathrm{H}\right] /\left[\mathrm{HD}^{+}+\mathrm{D}\right]=2.8 \pm 0.4$ and $2\left[\mathrm{H}^{+}+\mathrm{D}_{2}^{+}\right] /\left[\mathrm{D}^{+}+\mathrm{HD}^{+}\right]$ $=2.1 \pm 0.1$ on average across a broad range of electron impact energies. They noted that, in general, there tends to be a preference for ejection of the lightest nuclear fragment, namely the $\mathrm{H}$ nucleus in the above examples. Qualitatively, they discussed this in terms of the steepness of the potential energy surfaces for the different reaction pathways, indicating that steeper (more repulsive) surfaces favoured ejection of the lightest fragment. Furthermore, they supported this argument for ejection of the lightest 
fragment using classical reasoning in terms of a potential scattering description.

In this work, we use a crossed-beams coincidence 3D momentum imaging method $[21,43,44]$ that is particularly well suited for uniquely identifying the many different fragmentation channels of $\mathrm{D}_{2} \mathrm{H}^{+}$(see figure 1 ) that may be categorized as:

dissociation

$$
\begin{aligned}
\mathrm{D}_{2} \mathrm{H}^{+}+n \omega & \rightarrow \mathrm{H}^{+}+\mathrm{D}_{2} \\
& \rightarrow \mathrm{D}^{+}+\mathrm{HD} \\
& \rightarrow \mathrm{D}_{2}^{+}+\mathrm{H} \\
& \rightarrow \mathrm{HD}^{+}+\mathrm{D} \\
& \rightarrow \mathrm{H}^{+}+\mathrm{D}+\mathrm{D} \\
& \rightarrow \mathrm{D}^{+}+\mathrm{D}+\mathrm{H}
\end{aligned}
$$

single ionization

$$
\begin{aligned}
\mathrm{D}_{2} \mathrm{H}^{+}+n \omega & \rightarrow \mathrm{H}^{+}+\mathrm{D}_{2}^{+}+e^{-} \\
& \rightarrow \mathrm{D}^{+}+\mathrm{HD}^{+}+e^{-} \\
& \rightarrow \mathrm{H}^{+}+\mathrm{D}^{+}+\mathrm{D}+e^{-} \\
& \rightarrow \mathrm{D}^{+}+\mathrm{D}^{+}+\mathrm{H}+e^{-}
\end{aligned}
$$

and double ionization

$$
\mathrm{D}_{2} \mathrm{H}^{+}+n \omega \rightarrow \mathrm{H}^{+}+\mathrm{D}^{+}+\mathrm{D}^{+}+2 e^{-}
$$

where $n \omega$ denotes the interaction with the strong laser field. By applying our coincidence imaging technique, each of these channels is distinctly separated as illustrated in figure 1. The left panel, figure 1(a), shows raw data for the coincidence time-of-flight of two fragments hitting the detector ( $t_{2}$ arriving after $\left.t_{1}\right)$ within the same laser shot, accumulated over many laser shots. Labels identify the different breakup channels, with the first two fragments in the label determining the position in the spectrum. The two-body channels appear as sharp lines due to momentum conservation while the three-body channels appear broader, in this view, as the third fragment will carry a portion of the momentum. The unambiguous identification of the three-body channels can be better viewed by plotting the time-of-flight of the centre of mass of the first two fragments $\left(t_{12}\right)$ against that of the third fragment $\left(t_{3}\right)$, as shown in the right panel, figure 1(b), where the three-body channels now appear as sharp lines. While raw data is presented in figure 1, after applying momentum conservation, the false correlations distributed throughout the plot are discriminated against as shown, for example, in figure 2 of Ref. [23].

Another important feature of the coincidence 3D momentum imaging method is that it provides kinematic information on the fragments, allowing us to observe any 

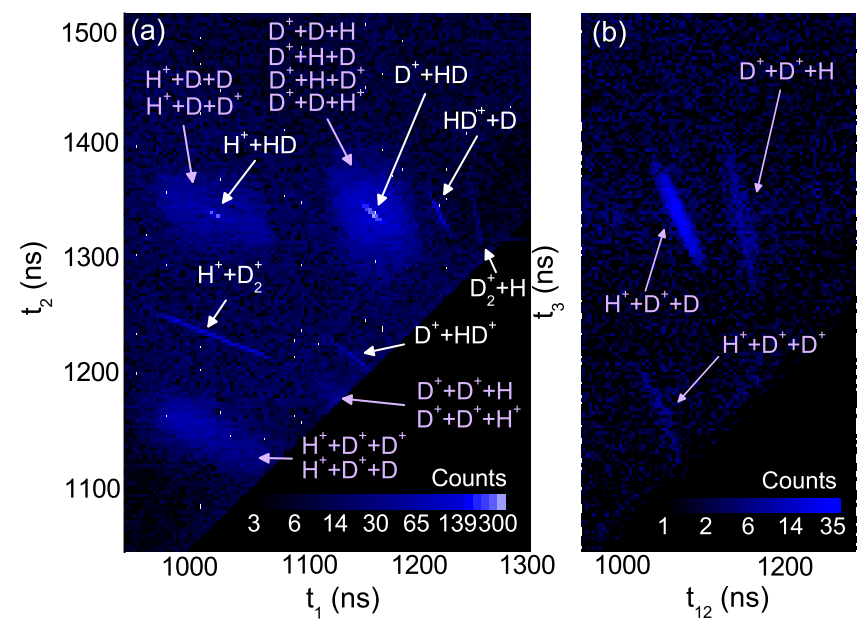

Figure 1. Coincidence time-of-flight maps showing raw data for $\mathrm{D}_{2} \mathrm{H}^{+}$breakup illustrating the separation of fragmentation channels. See text.

dynamic differences between channels. Details of the method applied to $\mathrm{D}_{3}^{+}$beams are presented elsewhere $[20,21,22,23,24]$ with a brief overview specific to $\mathrm{D}_{2} \mathrm{H}^{+}$to follow.

The $\mathrm{D}_{2} \mathrm{H}^{+}$ions were produced in an electron cyclotron resonance (ECR) ion source through fast-electron-impact ionization of $\mathrm{H}_{2}$ and $\mathrm{D}_{2}$ followed by the collision reactions, $\mathrm{H}_{2}^{+}+\mathrm{D}_{2}$ (proton transfer) and $\mathrm{D}_{2}^{+}+\mathrm{H}_{2}$ (atom transfer), both leading to $\mathrm{D}_{2} \mathrm{H}^{+}+\mathrm{H}$ products. After acceleration to $10 \mathrm{keV}$, the momentum-selected $\mathrm{D}_{2} \mathrm{H}^{+}$ion beam was transported to the laser interaction region. There, it was crossed at $90^{\circ}$ with $7 \mathrm{fs}(40 \mathrm{fs})$, $790 \mathrm{~nm}$ laser pulses focussed to a peak intensity of $10^{16} \mathrm{~W} / \mathrm{cm}^{2}\left(5 \times 10^{15} \mathrm{~W} / \mathrm{cm}^{2}\right)$.

In the interaction region, the ionic fragments from the breakup of $\mathrm{D}_{2} \mathrm{H}^{+}$were accelerated toward a detector downstream by an electrostatic field, applied using a longitudinal spectrometer. This enabled the neutral and ion fragments to be distinguished by their time-of-flight to the detector, which depends on the charge-toenergy ratio. The detector not only recorded the time-of-flight but also the position of the fragments on the detector. The fragments were detected in coincidence and the data were recorded in event mode allowing the three-dimensional momentum vector of each recorded fragment to be deduced. For two-body fragmentation of $\mathrm{D}_{2} \mathrm{H}^{+}$, a two-particle coincidence was required, and for three-body breakup, a three-particle coincidence was required. In essence, kinematically-complete information for the nuclear fragments of molecular breakup were recorded, but not the electrons from ionization channels.

The KER distributions for each of the channels obtained by this method using $7 \mathrm{fs}$ pulses are plotted in figure 2 with the exception of the three-body dissociation channels, $\mathrm{H}^{+}+\mathrm{D}+\mathrm{D}$ and $\mathrm{D}^{+}+\mathrm{D}+\mathrm{H}$, where the dissociation rates are extremely low (as is also the case for $\mathrm{D}_{3}^{+}[20]$ ). The figure panels are arranged to compare the distributions of channels where an isotopic effect might arise, and some plots include a statistical weighting factor - for example, the $\mathrm{D}^{+}+\mathrm{HD}$ channel is statistically twice as probable as the $\mathrm{H}^{+}+\mathrm{D}_{2}$ channel, hence the counts for the $\mathrm{H}^{+}+\mathrm{D}_{2}$ channel have been scaled by a 


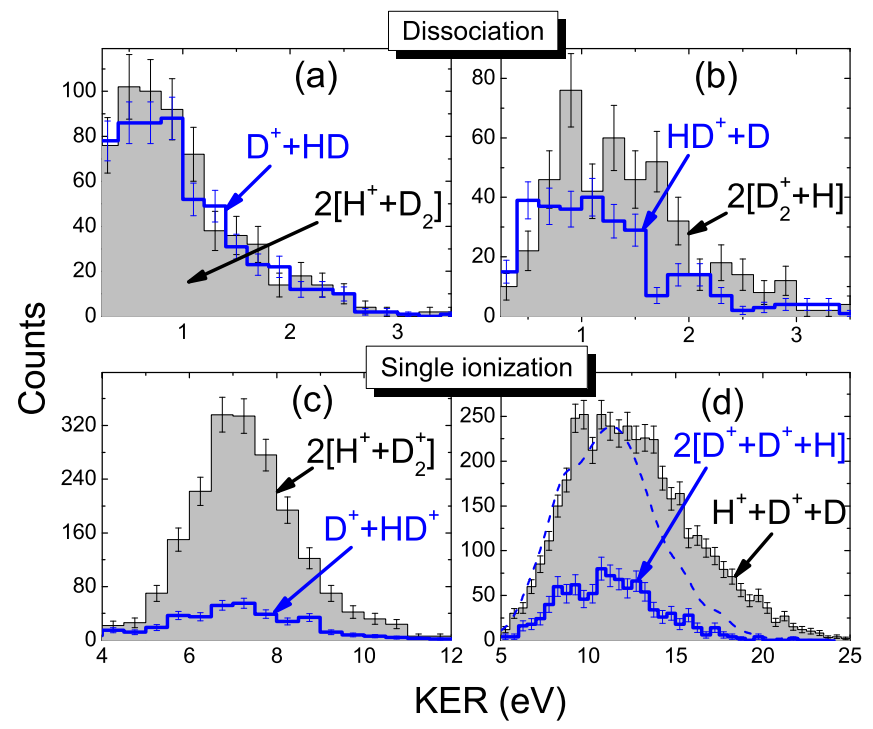

Figure 2. KER distributions for two-body dissociation (a,b) and single ionization $(\mathrm{c}, \mathrm{d})$ of $\mathrm{D}_{2} \mathrm{H}^{+}$using $7 \mathrm{fs}, 790 \mathrm{~nm}$ laser pulses at $10^{16} \mathrm{~W} / \mathrm{cm}^{2}$. The dashed line in (d) is a smoothed curve of the $\mathrm{D}^{+}+\mathrm{D}^{+}+\mathrm{H}$ channel arbitrarily scaled to the peak of the $\mathrm{H}^{+}+\mathrm{D}^{+}+\mathrm{D}$ channel for comparison. Error bars are determined from the statistical uncertainty in the data. Note that these plots are the measured data and do not account for the detection efficiencies of fragments as factored into the ratios in figure 3 (where the scaling factor for $2\left[\mathrm{H}^{+}+\mathrm{D}_{2}\right] /\left[\mathrm{D}^{+}+\mathrm{HD}\right]$ is $1.20 \pm 0.03$, for $2\left[\mathrm{D}_{2}^{+}+\mathrm{H}\right] /\left[\mathrm{HD}^{+}+\mathrm{D}\right]$ is $2.1 \pm 0.5$, for $2\left[\mathrm{H}^{+}+\mathrm{D}_{2}^{+}\right] /\left[\mathrm{D}^{+}+\mathrm{HD}^{+}\right]$is $1.22 \pm 0.02$, and for $\left[\mathrm{H}^{+}+\mathrm{D}^{+}+\mathrm{D}\right] / 2\left[\mathrm{D}^{+}+\mathrm{D}^{+}+\mathrm{H}\right]$ is $\left.0.6 \pm 0.2\right)$.

factor of two for comparison purposes. By integrating the total number of counts across the complete KER range for each channel, the isotopic ratios are obtained and presented in figure 3. For each of these ratios we have factored in the different detection efficiencies of the fragments (determined from our measurements) which for some of the channels can lead to significant uncertainties as displayed by the red error bars on the left side of each column. The statistical uncertainty in the data is also given by the purple error bars on the right side of each column. Recall that a ratio equal to 1 indicates no isotopic effect.

Summarizing the main results of this investigation (figure 3): we observe a large isotopic effect for the single ionization two-body breakup channels, where the $\mathrm{H}^{+}+\mathrm{D}_{2}^{+}$ channel dominates by more than a factor of five over the $\mathrm{D}^{+}+\mathrm{HD}^{+}$channel, using both 7 and $40 \mathrm{fs}$ pulses. Significant isotopic effects are also found at $7 \mathrm{fs}$ in the single ionization three-body channels, where $\mathrm{H}^{+}+\mathrm{D}^{+}+\mathrm{D}$ dominates $\mathrm{D}^{+}+\mathrm{D}^{+}+\mathrm{H}$, and dissociative twobody channels with $\mathrm{D}_{2}^{+}+\mathrm{H}$ dominating $\mathrm{HD}^{+}+\mathrm{D}$, but are almost absent at 40 fs for the same channels. In contrast, the dissociative two-body channels $\mathrm{H}^{+}+\mathrm{D}_{2}$ and $\mathrm{D}^{+}+\mathrm{HD}$ display only a small effect for either pulse duration. In what follows, we present an interpretation of these observations.

For single ionization, it was previously found that the ground state potential energy surface (PES) of the dication, in this case $\mathrm{D}_{2} \mathrm{H}^{2+}$, predominantly leads to two-body 
fragmentation, while the first excited state surface leads to three-body fragmentation [20]. For the ground state PES, shown in figure 4(a), there is a deep potential valley along increasing $R$ with $r$ fixed at $\sim 2.0$ a.u., which draws a propagating wavepacket on the surface towards it, favouring two-body breakup over three-body breakup (see the arrow directions).

An approach to visualizing any isotopic effect in fragmentation is to use massscaled coordinates, where the internuclear distances are scaled by the square-root of the reduced mass, $\sqrt{\mu}$, along that coordinate - see, for example, Ref. [45]. For breakup into $\mathrm{H}^{+}+\mathrm{D}_{2}^{+}$, this equates to scaling $R$ by $\sqrt{\mu}=\sqrt{4 m_{p} / 5}$ for stretching of the $\mathrm{H}^{+}-\mathrm{D}_{2}^{+}$ bond (and $r$ by $\sqrt{\mu}=\sqrt{m_{p}}$ for the $\mathrm{D}_{2}^{+}$stretch), where $m_{p}$ is the mass of the proton. For breakup into $\mathrm{D}^{+}+\mathrm{HD}^{+}, R$ is scaled by $\sqrt{\mu}=\sqrt{6 m_{p} / 5}$ for stretching the $\mathrm{D}^{+}-\mathrm{HD}^{+}$bond (and $r$ by $\sqrt{\mu}=\sqrt{2 m_{p} / 3}$ for the $\mathrm{HD}^{+}$stretch). This approach incorporates the effect of mass along a fragmentation coordinate into the potential, rather than the kinetic energy. That is, the kinetic energy no longer depends on mass.

In figure 4 , one can see that the potential along $R_{\text {scaled }}$ (i.e., $\sqrt{\mu} R$ ) drops faster for the $\mathrm{H}^{+}+\mathrm{D}_{2}^{+}$channel [figure $4(\mathrm{c})$ ] than the $\mathrm{D}^{+}+\mathrm{HD}^{+}$channel [figure $4(\mathrm{~d})$ ]. This is particularly evident from the cuts along the two-body reaction path shown in figure 4(e), where the potential of the $\mathrm{H}^{+}+\mathrm{D}_{2}^{+}$path is lowered with respect to the $\mathrm{D}^{+}+\mathrm{HD}^{+}$path (e.g., by $0.9 \mathrm{eV}$ at $R_{\text {scaled }}=6 \sqrt{m_{p}}$ a.u.) due to the scaling (visible, for example, by the steeper tangent for the $\mathrm{H}^{+}+\mathrm{D}_{2}^{+}$curve at the point marked + ). Hence, a nuclear wavepacket will favour the pathway leading to $\mathrm{H}^{+}+\mathrm{D}_{2}^{+}$over $\mathrm{D}^{+}+\mathrm{HD}^{+}$, in agreement with our observed ratio (6.0 at $7 \mathrm{fs}$ and 5.3 at $40 \mathrm{fs}$ ) for $2\left[\mathrm{H}^{+}+\mathrm{D}_{2}^{+}\right] /\left[\mathrm{D}^{+}+\mathrm{HD}^{+}\right]$in figure 3 . We note that this interpretation is harmonious with the classical formulation of Defrance et al. [42], which demonstrates that the timescale for fragmentation favours the channel that ejects the lighter nuclei, in this case the $\mathrm{H}^{+}+\mathrm{D}_{2}^{+}$channel.

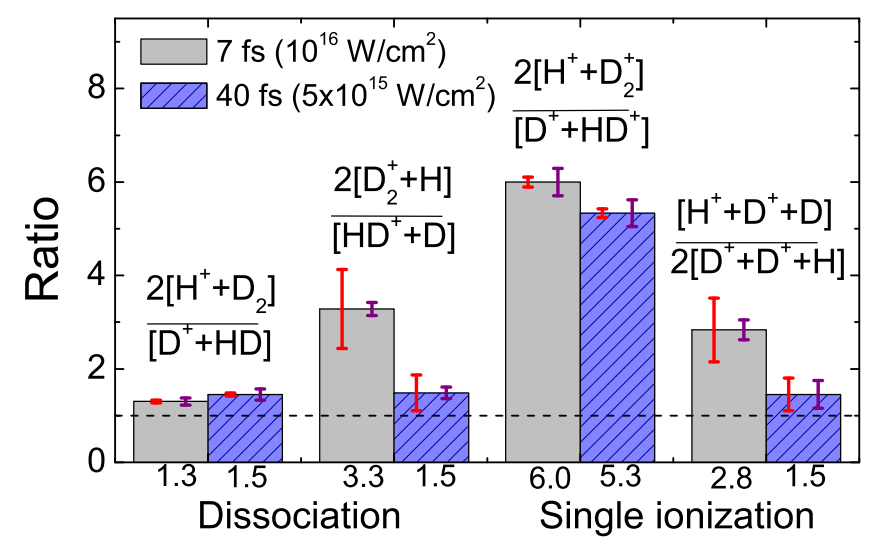

Figure 3. Isotopic ratios of channels for dissociation (left) and single ionization (right) using $7 \mathrm{fs}$ and $40 \mathrm{fs}, 790 \mathrm{~nm}$ laser pulses. The ratio values are given along the bottom of the columns. The red error bars on the left side of each column are due to detection efficiency uncertainties for the fragments while the purple error bars on the right side of each column are the statistical uncertainty in the data. 

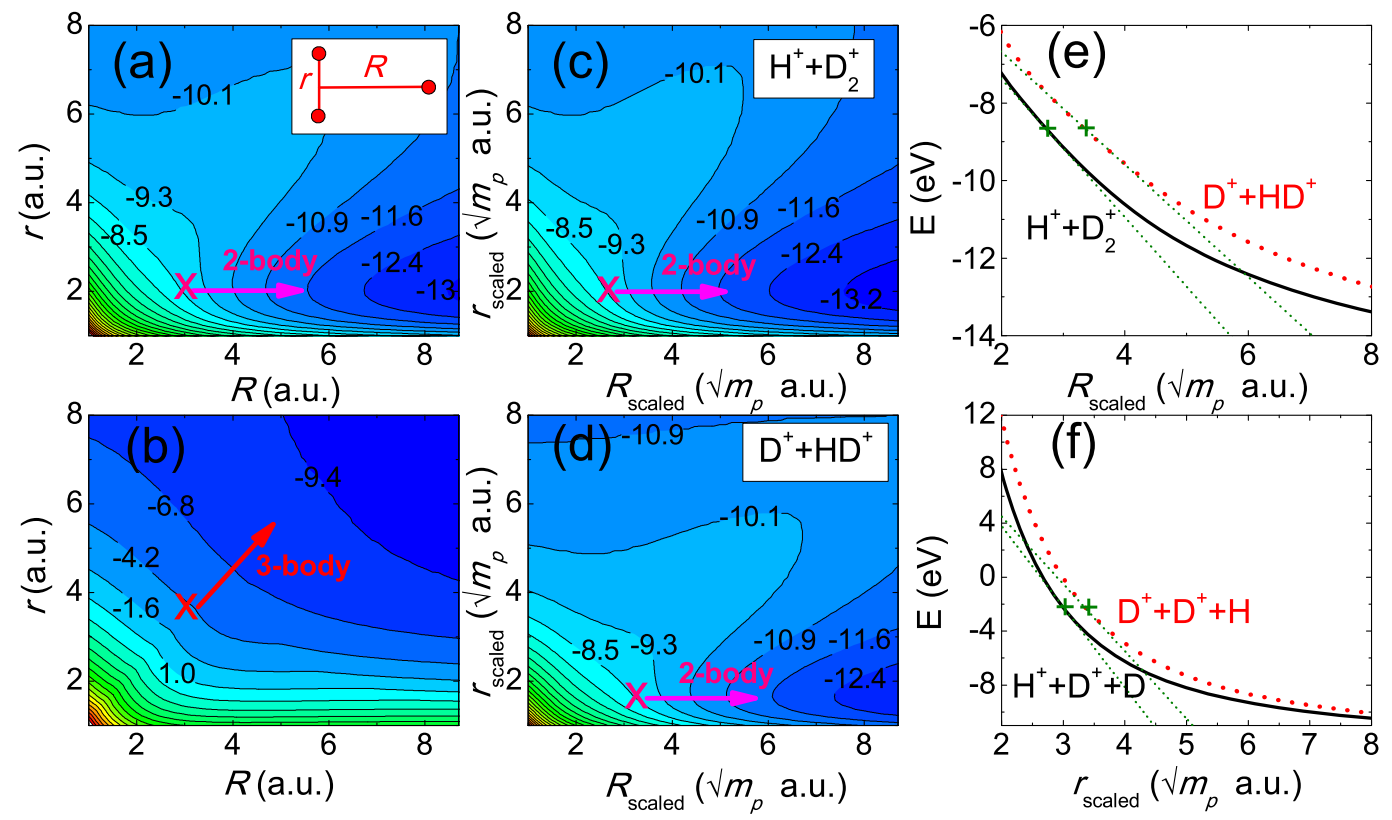

Figure 4. (a-b) Potential energy surfaces (PESs) calculated for $\mathrm{D}_{2} \mathrm{H}^{2+}$ by solving the Born-Oppenheimer equation in three dimensions using b-splines for (a) the ground state and (b) the first excited state. Contour energies are in units of eV. (c) and (d) are the same as (a) but with the internuclear coordinates scaled by the square-root of the reduced mass, $\sqrt{\mu}$, along that coordinate $\left(R_{\text {scaled }}\right.$ and $\left.r_{\text {scaled }}\right)$ - see text. The cross marks on $(\mathrm{a}-\mathrm{d})$ are an indication of where a nuclear wavepacket is likely to be excited to these surfaces while the arrows indicate the directions of two-body and three-body breakup as labelled. (e) shows cuts of the scaled ground state PES along $R_{\text {scaled }}$ with fixed $r=2.0$ a.u. for the $\mathrm{H}^{+}+\mathrm{D}_{2}^{+}$and $\mathrm{D}^{+}+\mathrm{HD}^{+}$channels. (f) shows cuts of the scaled first excited state PES along $r_{\text {scaled }}$ for the $\mathrm{H}^{+}+\mathrm{D}^{+}+\mathrm{D}$ and $\mathrm{D}^{+}+\mathrm{D}^{+}+\mathrm{H}$ channels, where the molecule fragments as an equilateral triangle. The thin dotted lines in (e) and (f), added as a visual aid, are tangents to the curves at points marked with + symbols. The inset of (a) defines the coordinates $R$ and $r$.

This interpretation neglects any effect of the laser field on the subsequent fragmentation dynamics once the nuclear wavepacket is promoted to the PES of the transient $\mathrm{D}_{2} \mathrm{H}^{2+}$. In general, we do not expect that the laser field will play a significant role in those dynamics, as ionization is expected to occur near the peak of the laser field (due to the high-intensity needed to ionize [20, 24]) and subsequent nuclear motion unfolds as the laser intensity becomes progressively weaker and less influential. Nevertheless, it is interesting to consider the possible effects of changing the laser intensity and/or pulse duration on the isotopic effect. In particular, with increasing intensity one can expect to ionize $\mathrm{D}_{2} \mathrm{H}^{+}$at smaller internuclear distance [24], where the PESs are steeper, thereby enhancing the isotopic effect. And for increasing pulse duration, ionization is expected to occur at larger internuclear distance [24] after the molecule has stretched in intermediate states, resulting in a decrease in the isotopic effect. While a comparison of the ratios for 7 fs pulses at $10^{16} \mathrm{~W} / \mathrm{cm}^{2}$ with $40 \mathrm{fs}$ pulses at $5 \times 10^{15} \mathrm{~W} / \mathrm{cm}^{2}$ is tentative at best, the general trend observed in figure 3 of a smaller 
isotopic effect for longer, lower intensity pulses does appear to follow.

One may attempt a similar interpretation for the three-body single ionization channels, $\mathrm{H}^{+}+\mathrm{D}^{+}+\mathrm{D}$ and $\mathrm{D}^{+}+\mathrm{D}^{+}+\mathrm{H}$. Three-body fragmentation is likely to mainly occur on the first excited state $\mathrm{D}_{2} \mathrm{H}^{2+}$ surface, shown in figure $4(\mathrm{~b})$, as deduced for $\mathrm{D}_{3}^{+}[20]$. A scaled cut of this potential for equilateral breakup of the molecule, shown in figure $4(\mathrm{f})$, indicates that the potential of the $\mathrm{H}^{+}+\mathrm{D}^{+}+\mathrm{D}$ path is lowered (e.g., by $0.5 \mathrm{eV}$ at $r_{\text {scaled }}=6 \sqrt{m_{p}}$ a.u.) with respect to the $\mathrm{D}^{+}+\mathrm{D}^{+}+\mathrm{H}$ path (and is steeper as seen by the tangent) in line with the fact that the $\mathrm{H}^{+}+\mathrm{D}^{+}+\mathrm{D}$ channel dominates at $7 \mathrm{fs}$ (figure 3).

For comparison purposes, the cuts of the potential we have taken are based on three-body breakup in the shape of an equilateral triangle. However, this is not entirely accurate, as revealed from a comparison of the different kinematics of these three-body channels. Figure 2(d) shows that for $7 \mathrm{fs}$ the $\mathrm{H}^{+}+\mathrm{D}^{+}+\mathrm{D}$ channel has a broader KER distribution than the $\mathrm{D}^{+}+\mathrm{D}^{+}+\mathrm{H}$ channel and, in particular, extends to higher KER values. The differences are emphasized when comparing the speed distributions of the individual fragments for the two channels as shown in figure 5. For the $\mathrm{D}^{+}+\mathrm{D}^{+}+\mathrm{H}$ channel, the final fragment speed distributions are all very similar showing that, as the molecular-ion breaks up, the $\mathrm{D}^{+}, \mathrm{D}^{+}$and $\mathrm{H}$ fragments all move apart approximately uniformly. In contrast, the $\mathrm{H}^{+}+\mathrm{D}^{+}+\mathrm{D}$ channel displays very different behaviour where the $\mathrm{H}^{+}$fragment moves considerably faster than the $\mathrm{D}^{+}$fragment, and in turn the $\mathrm{D}$ fragment. Hence, our results demonstrate that, in addition to the isotopic effects seen in the branching ratios, the total and individual fragment energies are also isotopically dependent.

Broadly, the interpretation by Defrance et al. [42], that isotopic effects can be associated with the steepness of the PES in the direction of fragmentation, is consistent with our data. That is, a flat PES will lead to no isotopic effect while a steep repulsive surface favours ejection of the lighter fragment. The KER distributions in figure 2 show that single ionization leads to high KER (above $6 \mathrm{eV}$ ), reflecting the steepness of the $\mathrm{D}_{2} \mathrm{H}^{2+}$ surfaces involved and thus why substantial isotopic effects in single ionization

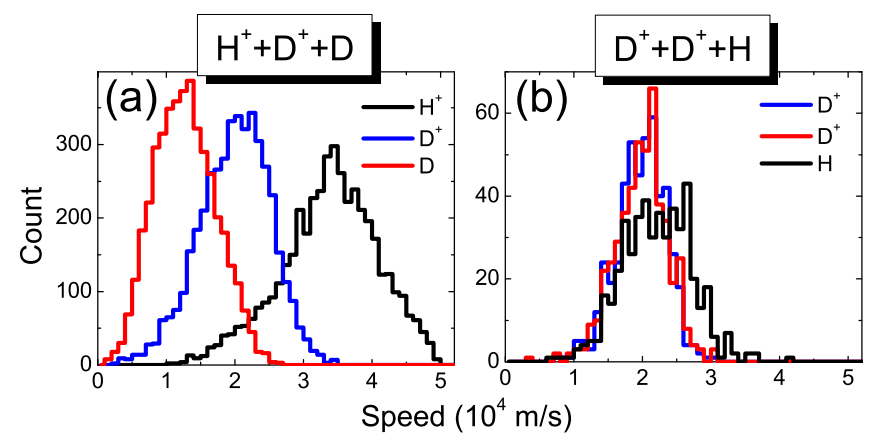

Figure 5. Speed distributions for the individual fragments of the (a) $\mathrm{H}^{+}+\mathrm{D}^{+}+\mathrm{D}$ and (b) $\mathrm{D}^{+}+\mathrm{D}^{+}+\mathrm{H}$ channels, using $7 \mathrm{fs}, 790 \mathrm{~nm}$ laser pulses at $10^{16} \mathrm{~W} / \mathrm{cm}^{2}$. In (b), the $\mathrm{D}^{+}$ nuclei are distinguished from one another by the order of their arrival at the detector. 
can be expected. Additionally, for two-body single ionization, it is the $\mathrm{H}^{+}+\mathrm{D}_{2}^{+}$channel that dominates in agreement with the prediction that ejection of the lightest nucleus is favoured. Furthermore, Defrance et al. found for dissociation of $\mathrm{D}_{2} \mathrm{H}^{+}$(following electron-impact) that the $2\left[\mathrm{D}_{2}^{+}+\mathrm{H}\right] /\left[\mathrm{HD}^{+}+\mathrm{D}\right]$ ratio displayed a significant isotopic effect while the $2\left[\mathrm{H}^{+}+\mathrm{D}_{2}\right] /\left[\mathrm{D}^{+}+\mathrm{HD}\right]$ ratio had no clear effect, due to the steepness of the PESs involved, again in line with our observations. The reduced isotopic effects we observe for $40 \mathrm{fs}$, at lower intensity, may also be due to this interpretation as excitation or ionization is traditionally found to occur at larger $R$ for longer lower-intensity pulses (e.g. [24]) where the potentials involved are not as steep.

Considering dissociation in more detail, unlike in electron-impact studies, discussing strong-field dissociation of $\mathrm{D}_{2} \mathrm{H}^{+}$in terms of the topology of a specific PES is complicated by the fact that the laser field can transfer population back and forth between different surfaces. Nevertheless, the relevant dissociation pathways have previously been identified for $\mathrm{H}_{3}^{+}$and $\mathrm{D}_{3}^{+}$studies under similar conditions as used here [23, 24]. Relating this knowledge to $\mathrm{D}_{2} \mathrm{H}^{+}$, there are likely to be two pathways (within the measured KER range), each leading to the $\mathrm{H}^{+}+\mathrm{D}_{2}$ and $\mathrm{D}^{+}+\mathrm{HD}$ channels with KER peaked at $\sim 0.6$ and $\sim 1.8 \mathrm{eV}$. Both pathways involve final transfer of the dissociative nuclear wavepacket onto the $\mathrm{X}^{1} A^{\prime}$ ground state at $R \sim 4.8$ a.u. $[23,24]$, where $R$ is the internuclear distance between the atomic and diatomic fragments (see definition in the inset of figure $4(\mathrm{a})$ ). For $R>4.8$ a.u., the $\mathrm{X}^{1} A^{\prime}$ state is relatively flat, which may explain the lack of a large isotopic effect for the $2\left[\mathrm{H}^{+}+\mathrm{D}_{2}\right] /\left[\mathrm{D}^{+}+\mathrm{HD}\right]$ ratio, across all $\mathrm{KER}$ (as deduced from figure $2(\mathrm{a}))$.

On the other hand, the $\mathrm{D}_{2}^{+}+\mathrm{H}$ and $\mathrm{HD}^{+}+\mathrm{D}$ channels involve dissociation on the excited $2^{1} A^{\prime}$ state. This state is repulsive and therefore more likely to produce an isotopic effect, as observed in electron-impact dissociation [42]. Specifically, the pathways involved at $7 \mathrm{fs}$ are expected to result in final excitation to the $2^{1} A^{\prime}$ state at $R \sim 4.8,3.8$ and 3.3 a.u. with KER values of about $0.6,1.0$ and $2.1 \mathrm{eV}$, respectively see $[23,24]$. The $2^{1} A^{\prime}$ state is more repulsive at smaller $R$, thus one can expect a bigger contribution of $\mathrm{D}_{2}^{+}+\mathrm{H}$ relative to $\mathrm{HD}^{+}+\mathrm{D}$ at larger $\mathrm{KER}$, i.e. ejection of the lighter nucleus is favoured for more repulsive potentials [42]. This is generally consistent with figure $2(\mathrm{~b})$, where indeed the ratio $2\left[\mathrm{D}_{2}^{+}+\mathrm{H}\right] /\left[\mathrm{HD}^{+}+\mathrm{D}\right]$ is greater with increasing KER (smaller $R$ ). We acknowledge, however, that such a discussion is over simplified as the nuclear wavepacket dynamics prior to final excitation to $2^{1} A^{\prime}$ can also play a role.

In summary, we have carried out the first strong-field measurements of the fundamentally important $\mathrm{D}_{2} \mathrm{H}^{+}$molecular ion. In doing so, we reveal a particularly strong preference for fragmentation to $\mathrm{H}^{+}+\mathrm{D}_{2}^{+}$over $\mathrm{D}^{+}+\mathrm{HD}^{+}$. Such isotopic effects in single ionization can be interpreted in terms of the shape of the $\mathrm{D}_{2} \mathrm{H}^{2+}$ PES using massscaled coordinates, and for two-body breakup agrees with the suggestion of Defrance et al. [42] that the channel which ejects the lighter particle is favoured. For dissociation, our results at $7 \mathrm{fs}$ also qualitatively agree with those of Defrance et al. for electronimpact, namely that the $2\left[\mathrm{D}_{2}^{+}+\mathrm{H}\right] /\left[\mathrm{HD}^{+}+\mathrm{D}\right]$ ratio displays a clear isotopic effect while the $2\left[\mathrm{H}^{+}+\mathrm{D}_{2}\right] /\left[\mathrm{D}^{+}+\mathrm{HD}\right]$ ratio does not. One would expect that theoretical treatment of 
this most simplistic, but still rather complex, polyatomic would elucidate its dynamics. These experimental results should serve as a benchmark for any such theory and help pave the way for exploring ever more complicated systems.

\section{Acknowledgements}

We thank Z. Chang's group for providing expertise with the laser beams and C. W. Fehrenbach for his help with the ion beams. This work was supported by the Chemical Sciences, Geosciences, and Biosciences Division, Office of Basic Energy Sciences, Office of Science, U.S. Department of Energy.

\section{References}

[1] Tennyson J 1995 Rep. Prog. Phys. 57421

[2] Oka T 2006 Proc. Natl. Acad. Sci. USA 10312249

[3] Geballe T R and Oka T 2006 Science 3121610

[4] Geballe T R and Oka T 1996 Nature 384334

[5] McCall B J, Geballe T R, Hinkle K H and Oka T 1999 Astrophys. J. 522338

[6] McCall B J, Geballe T R, Hinkle K H and Oka T 1998 Science 2791910

[7] McCall B J et al. 2003 Nature 422500

[8] Drossart P et al. 1989 Nature 340539

[9] Friedrich O, Alijah A, Xu Z and Varandas A J C 2001 Phys. Rev. Lett. 861183

[10] Carrington A and Kennedy R A 1984 J. Chem. Phys. 8191

[11] McNab I R 1995 Adv. Chem. Phys. 891

[12] Larsson M, McCall B J and Orel A E 2008 Chem. Phys. Lett. 462145

[13] Kokoouline V, Greene C H and Esry B D 2001 Nature 412891 and references therein

[14] Larsson M et al. 1993 Phys. Rev. Lett. 70430

[15] Sundström G et al. 1994 Science 263785

[16] Datz S, Sundström G, Biedermann C, Broström L, Danared H, Mannervik S, Mowat J R and Larsson M 1995 Phys. Rev. Lett. 74896

[17] Strasser D et al. 2001 Phys. Rev. Lett. 86779

[18] Hoshina K, Furukawa Y, Okino T and Yamanouchi K 2008 J. Chem. Phys. 129104302

[19] Kaziannis S, Liontos I, Karras G, Corsi C, Bellini M and Kosmidis C 2009 J. Chem. Phys. 131 144308

[20] McKenna J, Sayler A M, Gaire B, Johnson N G, Carnes K D, Esry B D and Ben-Itzhak I 2009 Phys. Rev. Lett. 103103004

[21] Sayler A M 2008 Measurements of Ultrashort Intense Laser-Induced Fragmentation of Simple Molecular Ions Ph.D. thesis Kansas State University

[22] McKenna J, Sayler A M, Gaire B, Johnson N G, Esry B D, Carnes K D and Ben-Itzhak I 2012 New J. Phys. 14103029

[23] Gaire B, McKenna J, Zohrabi M, Carnes K D, Esry B D and Ben-Itzhak I 2012 Phys. Rev. A 85 023419

[24] Sayler A M, McKenna J, Gaire B, Johnson N G, Esry B D, Carnes K D and Ben-Itzhak I 2012 Phys. Rev. A 86033425

[25] Alexander J D et al. 2009 J. Phys. B 42141004

[26] Lötstedt E, Kato T and Yamanouchi K 2011 Phys. Rev. Lett. 106203001

[27] Lötstedt E, Kato T and Yamanouchi K 2012 Phys. Rev. A 85053410

[28] Tchitchekova D S, Lu H, Chelkowski S and Bandrauk A D 2011 J. Phys. B 44065601

[29] Yu H, Zuo T and Bandrauk A D 1998 J. Phys. B 311533 
[30] Bandrauk A D and Ruel J 1999 Phys. Rev. A 592153

[31] Bandrauk A D, Chelkowski S and Kawata I 2003 Phys. Rev. A 67013407

[32] Lein M, Corso P P, Marangos J P and Knight P L 2003 Phys. Rev. A 67023819

[33] Bian X B, Peng L Y and Shi T Y 2008 Phys. Rev. A 78053408

[34] Yu H and Bandrauk A D 1997 Phys. Rev. A 56685

[35] Bandrauk A D and Yu H 1999 Phys. Rev. A 59539

[36] Kawata I, Kono H and Bandrauk A D 2001 Phys. Rev. A 64043411

[37] Yu H and Bandrauk A D 1995 J. Chem. Phys. 1021257

[38] McKenna J, Sayler A M, Gaire B, Johnson N G, Zohrabi M, Carnes K D, Esry B D and Ben-Itzhak I 2009 J. Phys. B 42121003

[39] Note that asymmetric breakup of small molecules in intense lasers has been observed in cases where the energies of the fragmentation channels differ significantly at the separated-atoms limit, e.g. $\mathrm{ND}^{+}[46], \mathrm{DCl}^{+}[47]$.

[40] Buhr H et al. 2010 Phys. Rev. A 81062702

[41] The factor of 2 on the numerator of the two-body ratios, for example, $2\left[\mathrm{H}+\mathrm{D}_{2}\right] /[\mathrm{D}+\mathrm{HD}]$, is a statistical weighting. That is, it is statistically twice as probable to form $\mathrm{D}+\mathrm{HD}$ as it is to form $\mathrm{H}+\mathrm{D}_{2}$, hence a ratio of $2\left[\mathrm{H}+\mathrm{D}_{2}\right] /[\mathrm{D}+\mathrm{HD}]=1$ indicates no isotopic effect.

[42] Defrance P, Jureta J J, Lecointre J and Urbain X 2011 J. Phys. B 44075202

[43] Ben-Itzhak I, Wang P Q, Xia J F, Sayler A M, Smith M A, Carnes K D and Esry B D 2005 Phys. Rev. Lett. 95073002

[44] Wang P Q, Sayler A M, Carnes K D, Xia J F, Smith M A, Esry B D and Ben-Itzhak I 2006 Phys. Rev. A 74043411

[45] Sayler A M, Leonard M, Carnes K D, Cabrera-Trujillo R, Esry B D and Ben-Itzhak I $2006 \mathrm{~J}$. Phys. B 391701

[46] McKenna J, Sayler A M, Gaire B, Johnson N G, Parke E, Carnes K D, Esry B D and Ben-Itzhak I 2008 Phys. Rev. A $\mathbf{7 7} 063422$

[47] Korolkov M V, Breunig H G and Weitzel K M 2007 Opt. Spectrosc. 103325 